\title{
Phase transitions and the internal noise structure of nonlinear Schrödinger equation solitons
}

\author{
M. J. Werner and S. R. Friberg \\ NTT Basic Research Laboratories, 3-1 Morinosato-Wakamiya, Atsugi-shi,Kanagawa-ken, \\ 243-01, Japan.
}

(October 22, 2018)

\begin{abstract}
We predict phase-transitions in the quantum noise characteristics of systems described by the quantum nonlinear Schrödinger equation, showing them to be related to the solitonic field transition at half the fundamental soliton amplitude. These phase-transitions are robust with respect to Raman noise and scattering losses. We also describe the rich internal quantum noise structure of the solitonic fields in the vicinity of the phase-transition. For optical coherent quantum solitons, this leads to the prediction that eliminating the peak side-band noise due to the electronic nonlinearity of silica fiber by spectral filtering leads to the optimal photon-number noise reduction of a fundamental soliton.
\end{abstract}

42.50.Ar, 42.50.Dv, 42.50.Lc , 42.65.Tg , 42.81.Dp 
An initially localized wavepacket oscillates or "breathes" as it evolves into a soliton in a system described by a nonlinear Schrödinger equation. For the special case of integral multiples of the fundamental soliton amplitude, this oscillation is periodic. Otherwise, the wavepacket asymptotically evolves into an integral order "higher-order" soliton or if the initial wavepacket energy is near that of a fundamental soliton, the oscillatory breathing motion decays. The underlying physics is that the spectrum broadens due to the self-phase modulation and the dispersion acts as a feedback mechanism to redirect energy to the pulse center.

A characteristic signature of soliton formation for pulses more energetic than the fundamental soliton (that is, for $N>1$ ) is the bifurcation and rejoining that occurs in the wavepacket spectral domain. Breathing oscillations occur for $N<1$ but no distinct bifurcation in the intensity spectrum occurs. (Note, we take $N=1$ as the amplitude of a fundamental soliton.) When bright solitons do not exist (e.g., for normal dispersion), there is no feedback mechanism and the energy is transported away from the pulse center. Similarly, for $N<0.5$ in the anomalous dispersion regime, no soliton emerges in the asymptotic field. An important property of the soliton system is that for $N>0.5$ the asymptotic field contains one or more fundamental solitons [ [1]. The nonlinear Schrödinger equation therefore predicts a phase-transition in the asymptotic field at $N=0.5$ from $0 \rightarrow 1$ solitons. The soliton physics which leads to this behavior has important consequences which have not been previously explored. For example, below the phase-transition, quantum effects are small, whereas above the transition, they play an important role in structuring the noise properties of optical pulses.

Recent quantum noise experiments with optical solitons in silica fiber used coherent mode-locked laser pulses as their source [2]. Though the pulses contained macroscopically large numbers of photons, clear and unambiguous quantum effects were found in the noise measurements. These arose as the initial solitonic fields contained shot-noise fluctuations that were incorporated into the soliton and the accompanying dispersive radiation. In the initial propagation regime, where quantum soliton noise experiments to now have been 
carried out, the interference between the emergent soliton and dispersive radiation figures significantly in the difference between classical and quantum soliton field theories. For example, quantum mechanically, oscillations are necessarily present when the initial state is coherent even when classically they are absent, as when the initial pulse is an unperturbed fundamental soliton. So far, only one quantum noise experiment [3] has reported direct experimental consequences of these oscillation effects - the oscillations in the photon-number noise of the spectrally filtered solitons as the soliton energy is increased.

This Letter briefly describes some of the new physics that emerges from consideration of the quantum-mechanical effects of solitonic oscillatory behavior. Perhaps most significant is the phase-transition in the spectral intensity noise of the solitonic field accompanied by a bifurcation in its noise spectrum as the energy approaches that of a fundamental soliton. The signature of this transition is predicted to occur in the experimentally accessible regime of a few soliton periods, even at room-temperature where the Raman noise in silica fiber is important for typical picosecond and sub-picosecond pulse durations. We also detail aspects of the internal quantum noise structure of a propagating, oscillating soliton.

The nonlinear Schrödinger equation has been used in various forms for the study of Bose-Einstein condensation [4], Bose superfluids [5], and propagating coherent quantum solitons [3, [6] where the nonlinear Schrödinger equation governs the dynamics of the photon flux amplitude. Its quantum-mechanical description utilizes a normal-order representation of the quantum fields so that losses introduced by Rayleigh scattering and spectral filtering do not explicitly introduce extra noise into the equations at zero temperature. In the normal-ordered positive-P representation, the evolution takes on the familiar form of a zero-temperature ( $T=0 \mathrm{~K}$ ) Ito stochastic damped quantum nonlinear Schrödinger equation (QNLSE) [6] in a co-moving frame

$$
\frac{\partial \phi}{\partial \zeta}=\left[-\gamma-\frac{i}{2}\left(1 \pm \frac{\partial^{2}}{\partial \tau^{2}}\right)+i \phi^{\dagger} \phi+\sqrt{i} \Gamma(\zeta, \tau)\right] \phi,
$$

where $\Gamma$ is a real Gaussian noise with zero mean and a correlation $\left\langle\Gamma(\zeta, \tau) \Gamma\left(\zeta^{\prime}, \tau^{\prime}\right)\right\rangle=\delta(\zeta-$ $\left.\zeta^{\prime}\right) \delta\left(\tau-\tau^{\prime}\right) / \bar{n}, \bar{n}$ is a dimensionless photon number scale, and the length and time variables 
$(\zeta, \tau)$ are the scaled coordinates in a reference frame that moves with the propagating field at the group velocity of the center frequency of the soliton. For this equation and the corresponding Hermitian conjugate equation for $\phi^{\dagger}$, the length scales as $t_{0}^{2} /\left|k^{\prime \prime}\right|$, with $t_{0}$ the pulse width and $k^{\prime \prime}$ the fiber's group velocity dispersion. Quantum field propagation is performed numerically [10] using the Raman modified quantum nonlinear Schrödinger equation in the positive-P representation [11, 12]. The output field photon number (scaled to the photon-number scale $\bar{n}$ which for results presented later is $10^{8}$ ) is, after spectral filtering

$$
\langle\hat{n}\rangle=\int d \omega f^{*}(-\omega) f(\omega)\langle\hat{I}(\omega)\rangle
$$

where $\hat{I}(\omega)=\hat{\phi}^{\dagger}(-\omega) \hat{\phi}(\omega)$ and $f(\omega)$ is the spectral filter function [14], specifically an ideal pass-band filter in the following calculations.

If one were to measure the spectral intensity noise of the output solitonic field, it would reveal a bifurcation or splitting of the noise spectrum as the input energy is increased. We illustrate this effect in Fig. 1 by plotting the intensity spectrum variance for several input energies after 4 soliton propagation periods. The shot-noise level in all figures is at the zero level due to the normal-ordered representation, and an arbitrary scale has been used on the vertical axis to emphasize the spectral structure. The horizontal axis is scaled to the soliton pulse width $t_{0}$ so that the noise structure is independent of the soliton pulse duration within the limit of applicability of the unmodified QNLSE (for example, no Raman effect). In addition to the classical bifurcation in the spectrum for $N>1$, the soliton system displays a bifurcation in the quantum noise spectrum for $N<1$. The transition from a single noise peak at $N=0.7$ to sub-shot noise fluctuations and a split noise spectrum for $N=0.9$ can be revealed by photon-number noise measurements after spectral filtering. At fundamental soliton energies $(N=1)$, the noise spectrum contains excess noise in the side peaks similar to $N=0.9$, shot-noise fluctuations at zero frequency offset and sub-shot noise fluctuations in between.

Optimizing the quantum noise reduction at each energy by varying both the spectral filter 
bandwidth and propagation distance over the interval $\xi \in\left(0, \xi_{\max }=4\right]$ ( $\xi$ is given in units of soliton periods) reveals a phase transition in the quantum noise correlations of the solitonic field. This transition depends on the maximum propagation distance as shown in Fig. 2 where it is depicted for $\xi_{\max }=4,8$. Optimization gives the maximal quantum correlation expected for the solitonic field and therefore reflects the internal quantum noise structure. The filter cut-off frequency varies from $1 / 10 t_{0}$ within the transition region to $3 / 8 t_{0}$ below the transition for $\xi_{\max }=4$. Note that for $\xi_{\max }=8$, the transition occurs at slightly lower energies as expected for longer propagation distances. In contrast, increasing the fiber losses moves the phase transition to higher energies. Figure 2 also shows the transition to be robust with respect to Raman noise and the intrinsic scattering losses of a silica fiber (assuming $T=300 K, \gamma=0.0236$, and $\xi_{\max }=4$ ). In the normal dispersion regime (where no feedback or breathing oscillations occur), wavepackets with the same parameters only show a gradual linear decrease in noise as the energy increases in the vicinity of the soliton phase-transition. Consequently, in the normal dispersion regime the optimal for all energies shown in Fig. 2 is at $\xi=4$. The optimized quantum-noise reduction in the normal dispersion case always displays sub-shot noise photon-number fluctuations, whereas the soliton remains at the shotnoise level below the transition. Our experimental demonstration of soliton squeezing [3], showed that lower energy pulses exhibited excess noise after spectral filtering. However, the results presented here show that excess noise for sub-fundamental soliton energies is not optimal. Summarizing, the classical $0 \rightarrow 1$ soliton transition at $N=0.5$ in the asymptotic field manifests itself in the quantum noise for propagation distances of a few soliton periods. This represents a new feature for systems described by the nonlinear Schrödinger equation.

The quantum noise spectral structure shown in Fig. 3 that develops with the propagation of fundamental solitons illustrates the striking difference between classical and quantum descriptions, even for photon numbers as large as $10^{8}$. Classical theory says that fundamental solitons only undergo a global phase shift as they propagate. However, Fig. 3 shows that their intensity noise spectrum undergoes considerable change even after only a few soliton periods, assuming a coherent $\operatorname{sech}\left(t / t_{0}\right)$ input pulse [15. The internal noise structure 
of the propagating solitonic field, which can be probed by measuring the photon number fluctuations of spectrally filtered pulses, provides new insights into soliton dynamics. One important unexpected new feature is the production of excess noise in the spectral sidebands that peaks at side-band frequencies of $0.125 / t_{0} \mathrm{~Hz}$. This excess noise, shown in red in Fig. 33, can easily be removed by a spectral filter. Accompanying this excess noise are regions of sub-shot noise fluctuations, shown as dark blue in Fig. 迸, which are correlated with the excess noise.

The photon-number squeezing and the phase-transition is perhaps most easily explained by visualizing the solitons internal quantum noise structure and considering the effect of a spectral filter. Experimentally, the quantum correlations between different components of the noise can be determined by varying the spectral filter bandwidth and center frequency while measuring the changing noise variance of the transmitted energy. Figure 4 shows the changes in the photon number statistics of filtered fundamental solitons resulting from changing the filter bandwidth and propagation distance. For a wide filter bandwidth (i.e., much larger than $0.125 / t_{0} \mathrm{~Hz}$ ), the overall noise is near the shot-noise level. Decreasing the filter bandwidth reduces the noise level to a minimum and then increases it again as the

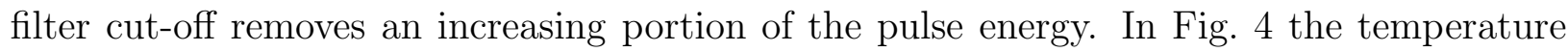
of the phonon reservoir is $300 \mathrm{~K}$ and $t_{0}=1 \mathrm{ps}$. Over the first four soliton periods, there is a clear optimum for the choice of filter cut-off frequency: $125 \pm 12.5 \mathrm{GHz}$ at a propagation distance of $3 \pm 0.2$ soliton periods. This is exactly the same frequency as Fig. 目 for the peak of the spectral intensity noise variance due to the electronic nonlinearity. As Raman effects are included, there is an asymmetry in the spectral intensity noise spectrum and an increase in the spectral intensity fluctuations with increasing temperature. Despite this effect, Fig. 1 shows significant photon-number squeezing up to 4.8dB below shot-noise, even at room-temperature.

The noise spectrum for a pulse with slightly more energy than a fundamental soliton has a different noise structure than that of a fundamental soliton. This can lead to dramatic changes in the measured noise levels after spectral filtering. Figure 5 shows the noise spec- 
trum when $N=1.1$, clearly showing excess noise near the center frequencies, in constrast to the $N=1$ case (Fig. 3) where the fluctuations were at or below shot-noise level [18]. This leads to large excess noise levels for strongly filtered pulses, which was observed experimentally [3]. Despite the small increase in input energy (21\%), the resulting quantum noise properties are very different and this illustrates the importance of understanding the complexity of the quantum noise structure of these solitonic fields.

Summarizing, we predict a phase-transition in systems governed by nonlinear Schrödinger equations detectable by measuring the particle number fluctuations in a selected subspace of the total Hamiltonian. The choice of a sub-space corresponding to the spectral filtering of modes farthest away from the slowly-varying envelope carrier frequency leads to a transition in the filtered pulse photon-number fluctuations which corresponding to the phase-transition in the quantum noise of the propagating solitonic field. (This squeezing transition in the co-moving frame, incidentally, has similarities to evaporative cooling in Bose-Einstein condensation.) We further showed that this transition is related to the classical transition to soliton propagation that occurs at $N=0.5$ and to the bifurcation in the intensity noise spectrum, and that it can be seen at easily accessible propagation distances. The internal quantum noise structure of the soliton field was shown to differ qualitatively depending on whether soliton energies were above or below that of the phase-transition and also a fundamental soliton. The optimal filter bandwidth for reducing photon-number fluctuations for fundamental solitons was found to correspond to removal of the peak in the electronic nonlinearity induced intensity noise spectral side-bands.

We have restricted our attention to systems near the $0 \rightarrow 1$ soliton phase-transition where Raman effects in silica fiber do not play a dominant role for picosecond pulses. However, higher-order nonlinearities and dispersion may effect the quantum noise propagation of shorter pulses and will be the subject of further studies. 


\section{REFERENCES}

[1] V. Zakarov and A. Shabat, Soviet Physics JETP 34, 62 (1972).

[2] P. D. Drummond, R. M. Shelby, S. R. Friberg and Y. Yamamoto, Nature 365, 307 (1993); Hermann A. Haus and William S. Wong, Rev. Mod. Phys. 68, 423 (1996).

[3] S. R. Friberg, S. Machida, M. J. Werner, A. Levanon and Takaaki Mukai, Phys. Rev. Lett. 77, 3775 (1996).

[4] C. C. Bradley, C. A. Sackett, J. J. Tollet and R. G. Hulet, Phys. Rev. Lett. 75, 1687 (1995).

[5] C. Josserand, Y. Pomeau, and S. Rica, Phys. Rev. Lett. 75, 3150 (1995).

[6] S. J. Carter, P. D. Drummond, M. D. Reid and R. M. Shelby, Phys. Rev. Lett. 58, 1841 (1987).

[7] Y. Lai and H.A. Haus, Phys. Rev. A 40, 844 (1989); Phys. Rev. A 40, 854 (1989).

[8] F. X. Kaertner and H.A. Haus, Phys. Rev. A 48, 2361 (1993).

[9] M. J Werner, Phys. Rev. A. 54, R2567 (1996).

[10] M. J. Werner and P. D. Drummond, J. Computational Phys. 132, 312 (1997).

[11] S. J Carter and P. D. Drummond, Phys. Rev. Lett. 67, 3757 (1991).

[12] An effect at low frequencies is GAWBS noise [13] which differs significantly depending on the fiber used. For this reason no attempt is made to include GAWBS contributions explicitly although one of the advantages of the approach used here is that such details can be included easily for specific fibers.

[13] R. M. Shelby, M. D. Levenson and P. W. Bayer, Phys. Rev. Lett. 54, 939 (1995).

[14] An interesting point is that the Raman effect provides an effective filter mechanism on the dynamics of the nonlinear system and the noise added is temperature-dependent 
colored noise. The Raman gain extends out to $40 \mathrm{THz}$ but the main contribution extends only to about $20 \mathrm{THz}$. For very short pulses this may provide unexpected properties. This effective filter mechanism is quite general and arises from tracing out an interacting field whose coupling with the field of interest is strongly frequency dependent.

[15] It is expected from the earlier work of Gordon [16] on the perturbed classical NLSE that vacuum fluctuations in the input pulse will produce a modulation of the photon-number variance due to the dispersive radiation (or excited continuum). This can be seen by writing the ensemble averaged intensity dependent nonlinearity using the truncated Fokker-Planck equation for the Wigner representation [17]. A heuristic argument can be made that an initial coherent pulse (a Gaussian distribution) produces an effective increase in the nonlinearity due to the $1 / 2$ photon per mode vacuum fluctuations which modulate the intensity and in turn the spectrum.

[16] J. P. Gordon, J. Opt. Soc. Am. B 9, 91 (1990).

[17] P. D. Drummond and A. D. Hardman, Europhys. Lett. 21, 279 (1991).

[18] The simulations for all figures were carried out using a time window of 80ps which determines the frequency resolution to be $12.5 \mathrm{GHz}$. 


\section{FIGURES}

FIG. 1. Scaled intensity spectrum variance for $N=0.7,0.9,1.0$ at $\zeta=2 \pi$ using $\phi(0, \tau)=N \operatorname{sech}(\tau)$.

FIG. 2. The quantum noise phase transition as the input energy is increased depicted using the photon-number variance of the spectrally filtered solitonic field. The full line is the ideal QNLSE at $\xi=4$, the dotted line is the ideal QNLSE at $\xi=8$, the dashed line is at $\xi=4$ with the Raman interaction at $T=300 K$ with $0.1 \mathrm{~dB} / \mathrm{km}\left(0.0236 \mathrm{~dB} / z_{0}\right.$ for $\left.z_{0}=370 \mathrm{~m}\right)$ losses and dot-dashed line is the latter case in the normal dispersion regime.

FIG. 3. Scaled intensity spectrum variance versus propagation distance for $\phi(0, \tau)=\operatorname{sech}(\tau)$ initial input pulses using the ideal QNLSE.

FIG. 4. Photon-number variance versus propagation distance and spectral filter cut-off frequency for $N=1$ at $T=300 K, t_{0}=1$ ps.

$\begin{array}{llll}\text { FIG. } & 5 . & \text { Scaled } & \text { intensity }\end{array}$ variance versus propagation distance for $N=1.1, \phi(0, \tau)=N \operatorname{sech}(\tau)$, initial input pulses using the ideal QNLSE. By using the color map of Fig. 3, the min/max values are clamped. 


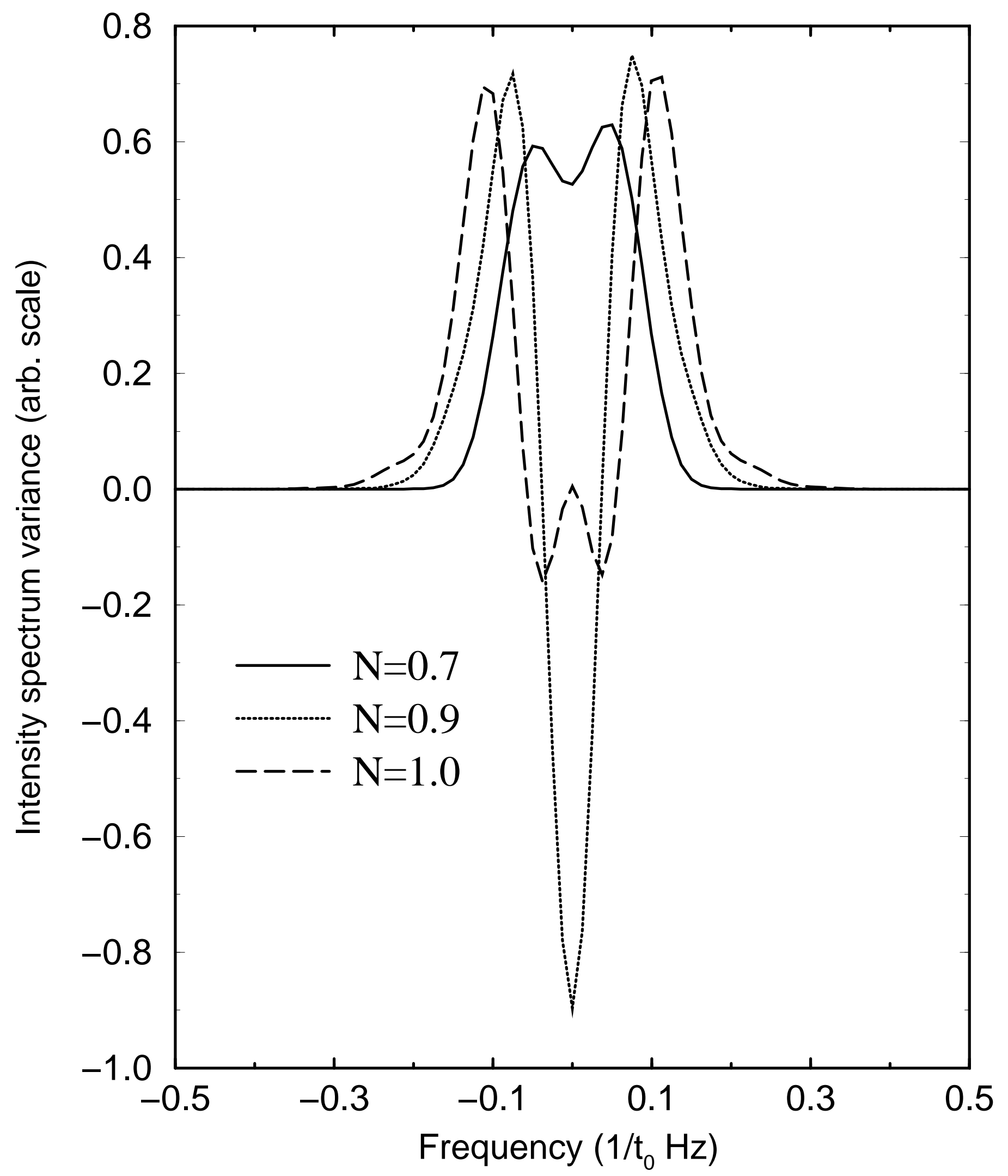




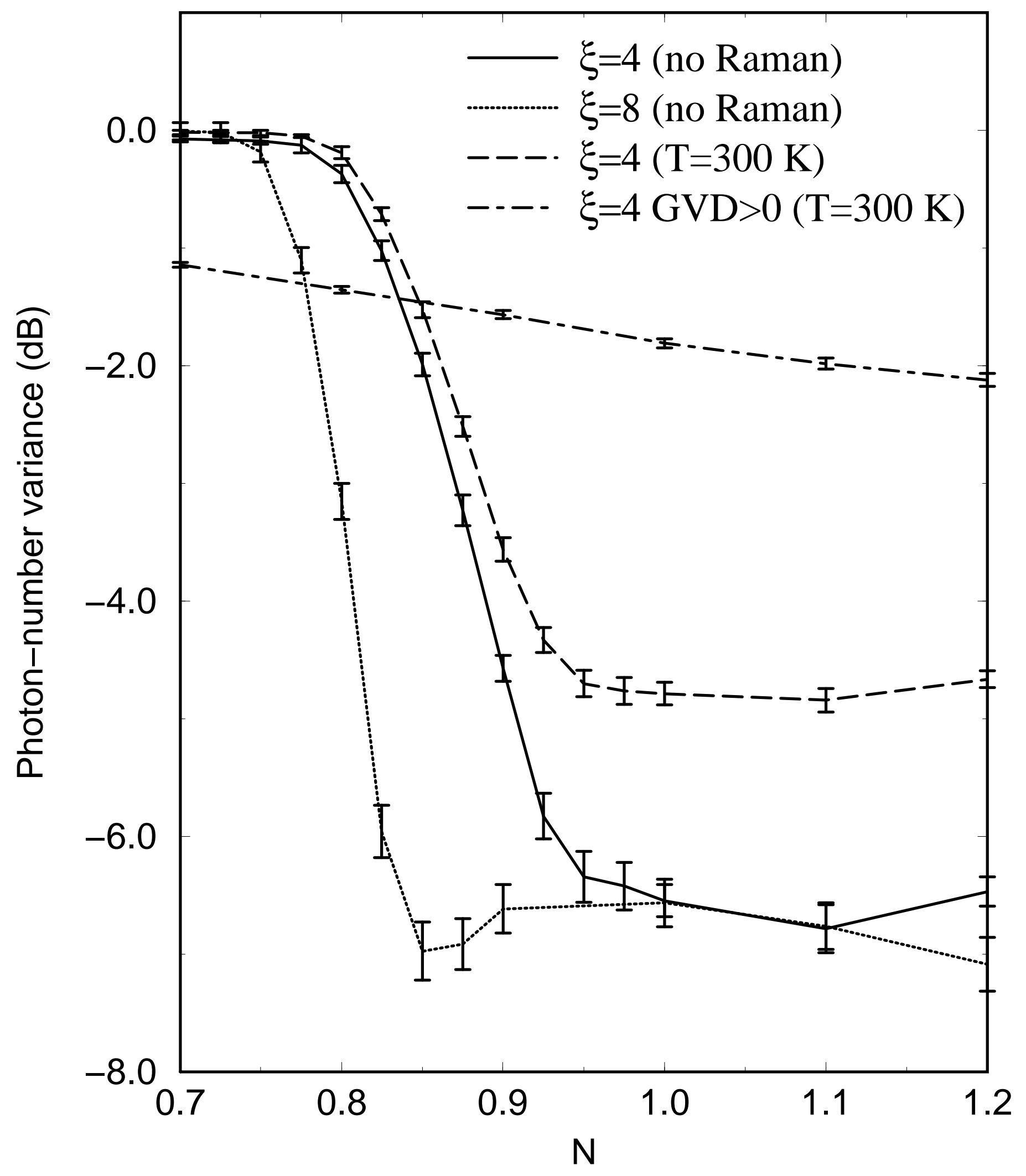



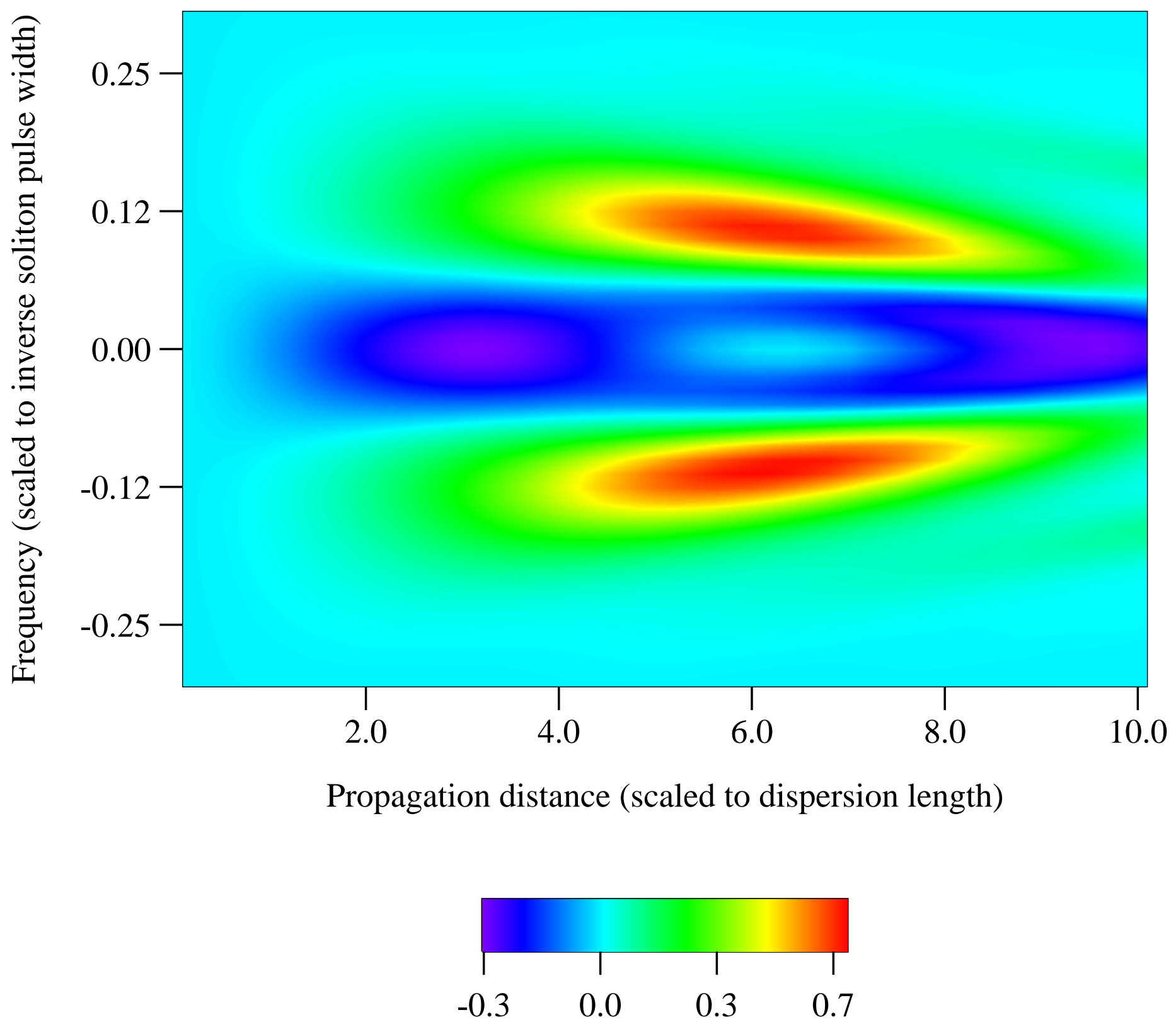

Spectral intensity variance (arb. scale) 


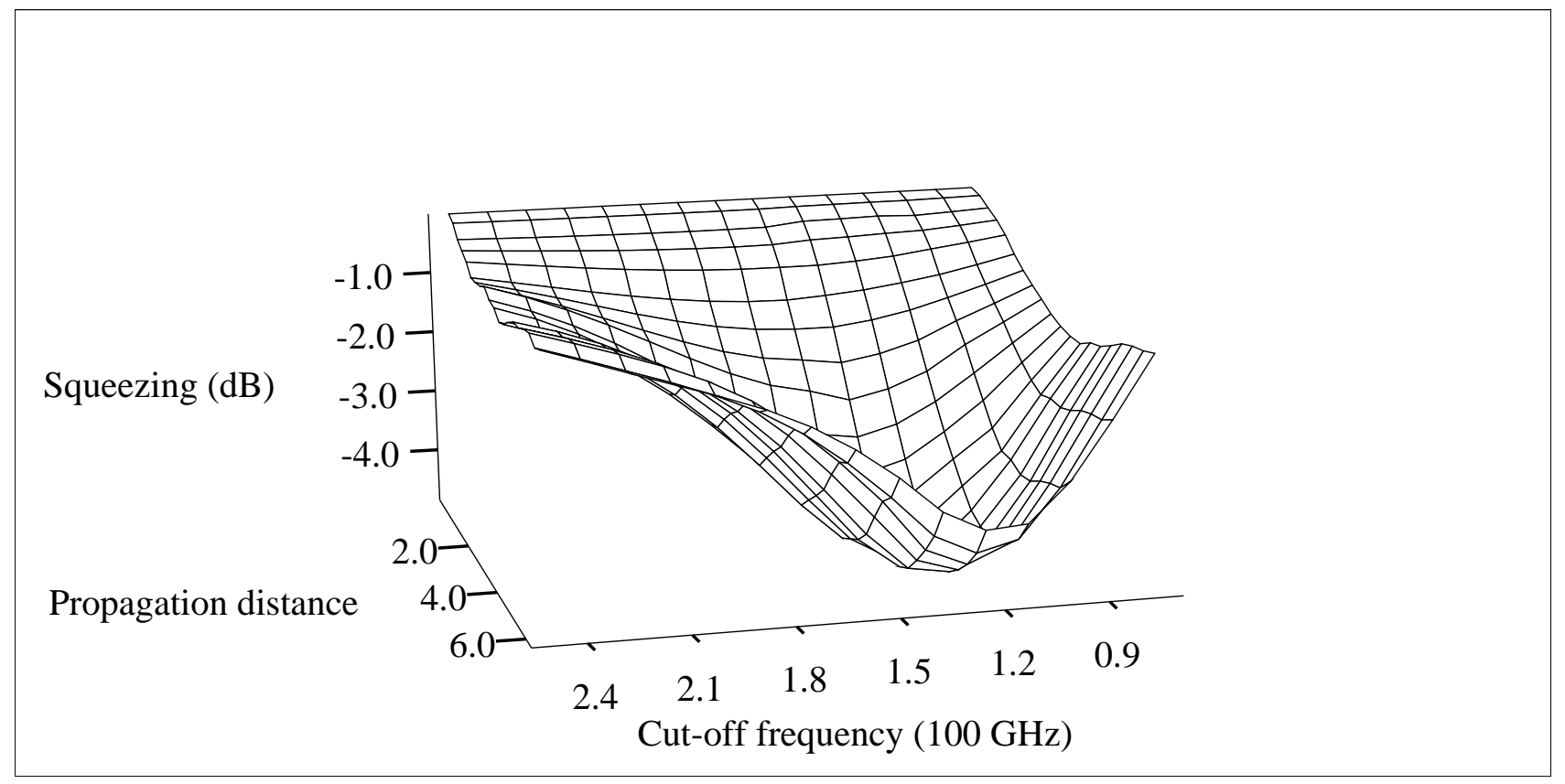



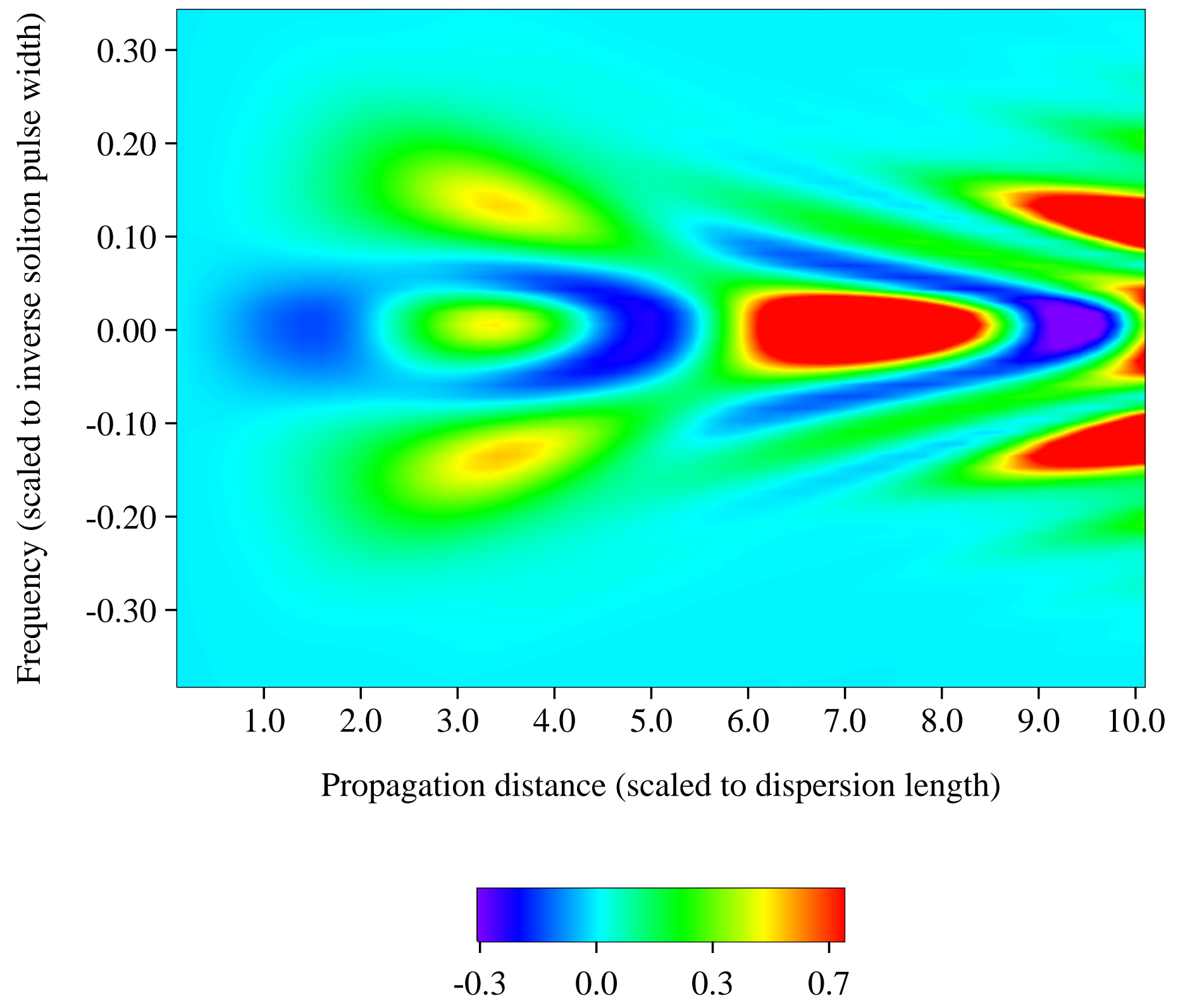

Spectral intensity variance (arb. scale) 